\title{
A HERO'S LITTLE HORSE: DISCOVERY OF A DISSOLVING STAR CLUSTER IN PEGASUS
}

\author{
Dongwon Kim and Helmut JerJen \\ Research School of Astronomy and Astrophysics, The Australian National University, Mt Stromlo Observatory, via Cotter Road, \\ Weston, ACT 2611, Australia; dongwon.kim@anu.edu.au, helmut.jerjen@anu.edu.au \\ Received 2014 August 19; accepted 2014 November 11; published 2015 January 15
}

\begin{abstract}
We report the discovery of an ultra-faint stellar system in the constellation of Pegasus. This concentration of stars was detected by applying our overdensity detection algorithm to the Sloan Digital Sky Survey Data Release 10 and confirmed with deeper photometry from the Dark Energy Camera (DECam) at the $4 \mathrm{~m}$ Blanco telescope. The best-fitting model isochrone indicates that this stellar system, Kim 1, features an old (12 Gyr) and metal-poor $([\mathrm{Fe} / \mathrm{H}] \sim-1.7)$ stellar population at a heliocentric distance of $19.8 \pm 0.9 \mathrm{kpc}$. We measure a half-light radius of $6.9 \pm 0.6 \mathrm{pc}$ using a Plummer profile. The small physical size and the extremely low luminosity are comparable to the faintest known star clusters Segue 3, Koposov 1 and 2, and Muñoz 1. However, Kim 1 exhibits a lower star concentration and is lacking a well-defined center. It also has an unusually high ellipticity and irregular outer isophotes, which suggests that we are seeing an intermediate mass star cluster being stripped by the Galactic tidal field. An extended search for evidence of an associated stellar stream within the $3 \mathrm{deg}^{2}$ DECam field remains inconclusive. The finding of Kim 1 is consistent with current overdensity detection limits and supports the hypothesis that there are still a substantial number of extreme low-luminosity star clusters undetected in the wider Milky Way halo.
\end{abstract}

Key words: galaxies: dwarf - globular clusters: general - Local Group

\section{INTRODUCTION}

Modern imaging surveys like the Sloan Digital Sky Survey (SDSS; York et al. 2000; Ahn et al. 2014) have significantly contributed to the discoveries of new stellar objects in the Milky Way (MW) halo including satellite galaxies (e.g., Willman et al. 2005; Belokurov et al. 2006; Irwin et al. 2007; Walsh et al. 2007) and star clusters (Koposov et al. 2007; Belokurov et al. 2010; Muñoz et al. 2012; Balbinot et al. 2013; Belokurov et al. 2014; Laevens et al. 2014). The new satellite galaxies are characterized by low luminosities $\left(-8 \lesssim M_{V} \lesssim-1.5\right.$; Martin et al. 2008) and low metallicities down to $[\mathrm{Fe} / \mathrm{H}]<-3$ (Kirby et al. 2008; Norris et al. 2010; Simon et al. 2011; Koch \& Rich 2014). The emergence of this new class of dwarf galaxies now questions previous ideas about the low mass limit of galaxy formation. The other new MW halo objects are star clusters with extremely low luminosities $\left(-2.5 \lesssim M_{V} \lesssim 0\right)$ and small halflight radii $(<10 \mathrm{pc})$. Globular clusters of such extreme nature are thought to be suffering stellar mass loss via dynamical processes such as tidal disruption or evaporation (Gnedin \& Ostriker 1997; Rosenberg et al. 1998; Koposov et al. 2007), and there is growing evidence based on observations to support this hypothesis (Carraro et al. 2007; Carraro 2009; NiedersteOstholt et al. 2010; Fadely et al. 2011). The discoveries of new ultra-faint star clusters in the Galactic halo will provide valuable resources for studies of their evolutionary phases as well as the formation history of the Galactic halo.

In this paper, we present the discovery of a new ultra-faint stellar system named Kim 1, in the constellation of Pegasus found in SDSS Data Release 10 (DR10) and confirmed with deep Dark Energy Camera (DECam) imaging (Sections 2 and 3). Its total luminosity is measured to be $\sim 0.3$ mag fainter than that of Segue $3\left(M_{V}=0.0 \pm 0.8\right)$ known to be probably the faintest star cluster to date (Fadely et al. 2011). Kim 1 lies at a heliocentric distance of approximately $20 \mathrm{kpc}$, is highly elongated $(\epsilon=0.42)$, and has a half-light radius of $\sim 7 \mathrm{pc}$
(Section 4). In Section 5, we discuss the possible origin of the stellar overdensity and draw our conclusions.

\section{DISCOVERY}

The SDSS imaging data are produced in the ugriz photometric bands to a depth of $r \sim 22.5 \mathrm{mag}$ (York et al. 2000). DR10 includes all the previous data releases, covering $14,555 \mathrm{deg}^{2}$ around the north Galactic pole (Ahn et al. 2014), and is publicly available on the SDSS-III Web site. ${ }^{1}$

We use an overdensity detection algorithm built upon the method of Walsh et al. (2009) to analyze the SDSS-DR10 point-source catalog. The algorithm has been designed to detect stars consistent with an old and metal-poor population, which reflects the typical characteristics of MW satellite galaxies (see, e.g., Kirby et al. 2013). At given heliocentric distance intervals we apply a photometric filter in color-magnitude space employing isochrone masks based on the Dartmouth models (Dotter et al. 2008) and the SDSS photometric uncertainties. We then bin the R.A., decl. positions of the selected stars into a $1.0 \times 1.0$ array with a pixel size between $18-25$ arcsec. This array is then convolved with a Gaussian kernel with a FWHM between $42-59$ arcsec. We empirically measure the statistical significance of potential overdensities by comparing their signal-to-noise ratios (S/Ns) on the array to those of random clustering in the residual Galactic foreground. This process is repeated by shifting the isochrone masks over a range of distance moduli $(m-M)$ from 16 to $22 \mathrm{mag}$, corresponding to the heliocentric distance interval $16<D<250 \mathrm{kpc}$, where the upper limit is the virial radius of the MW.

The detection algorithm successfully recovered all of the recently reported MW galaxy companions in the SDSS footprint as well as other resolved stellar overdensities such as Balbinot 1 (Balbinot et al. 2013). Our measured statistical clustering significances of the MW satellites, including Leo V and Bootes II

\footnotetext{
1 http://www.sdss3.org/dr10/
} 
that were reported as marginal detections by Walsh et al. (2009) and Koposov et al. (2008), were all at least $6.0 \sigma$ above the Poisson noise of Galactic stars while the significance of a newly found stellar overdensity reached $\sim 6.8 \sigma$ at its maximum. This object, which we chose to call Kim 1, was found at the location $22^{\mathrm{h}} 11^{\mathrm{m}} 41^{\mathrm{s}} .28,+07^{\mathrm{d}} 01^{\mathrm{m}} 31^{\mathrm{s}} .8(\mathrm{~J} 2000)$. It is also worth to mention that we detected a few more stellar concentrations over the entire SDSS-DR10 footprint with comparable significance but at larger distance moduli $(m-M)>20$. They will require deeper followup imaging observations to reach the main-sequence turnoff in the color-magnitude diagram (CMD) to confirm their identities.

\section{FOLLOW-UP OBSERVATIONS AND DATA REDUCTION}

To investigate the nature of Kim 1, we conducted deep followup observations using the DECam at the $4 \mathrm{~m}$ Blanco Telescope at Cerro Tololo Inter-American Observatory on 2014 July 17. DECam has an array of sixty-two $2 \mathrm{k} \times 4 \mathrm{k}$ CCD detectors with a 2.2 degrees field of view and a pixel scale of 0.27 (unbinned). We obtained a series of $8 \times 210 \mathrm{~s}$ dithered exposures in $g$ and $5 \times 210 \mathrm{~s}$ in $r$ band under photometric conditions. The mean seeing was $1^{\prime \prime} .0$ in both filters. The fully reduced and stacked images were produced by the DECam community pipeline (Valdes et al. 2014). We used WeightWatcher (Marmo \& Bertin 2008) for weight-map combination and SExtractor (Bertin \& Arnouts 1996) for source detection and photometry. Sources were morphologically classified as stellar or non-stellar objects. We cross-matched the extracted point sources with SDSS stars in our field of view using the STILTS software (Taylor 2006) with a $1^{\prime \prime}$.0 positional tolerance. The photometric calibration was restricted to stars in the magnitude range $19.25<r_{0}<21.25 \mathrm{mag}$ to stay below the saturation limit of our DECam images and above the $5 \sigma$ limit of the SDSS photometry. Finally, all magnitudes of the calibrated sources were extinction corrected with the Schlegel et al. (1998) extinction maps.

We performed artificial star tests to determine the photometric completeness as a function of magnitude. We use the starlist function in the IRAF package artdata to generate 1000 randomly distributed artificial stars for each $g$ and $r$ band in the range of $19<g, r<24$, and then employ the mkobjects function to inject them into the DECam images. We then extract the stars from the images using the same pipeline as we used on the original science images and cross-match all the point sources with the list of input artificial stars using STILTS to measure the recovery rate as a function of magnitude. Figure 1 shows the completeness levels for both photometric bands. The error bars for each bin are derived from the binomial distribution as in Bolte (1989). Overplotted are the best-fitting interpolation functions described in Fleming et al. (1995). We determine the $90 \%$ completeness levels of our photometry at $g=23.15$ and $r=22.80$, respectively.

Figure 2 shows the stellar overdensity in the running window after applying the photometric filter with a Dartmouth isochrone of $12 \mathrm{Gyr}$ and $[\mathrm{Fe} / \mathrm{H}]=-1.7$ (Dotter et al. 2008). The top left panel shows the sky positions of DECam stars fainter than the saturation limit $\left(r_{0} \approx 19 \mathrm{mag}\right)$ and brighter than the $90 \%$ completeness level $\left(r_{0}=22.80\right)$, passing the isochrone filtering process at the distance modulus $(m-M)=16.5 \mathrm{mag}$. The top right panel shows the spatial density plot with a pixel size 23".4 and convolved with a Gaussian kernel with a FWHM of 55".1, where the colors of the pixels represent the output signals $(s)$ of the convolution. The pixels above the foreground density with values $\tilde{s}+2.5 \sigma_{s} \approx 0.3$ are marked in yellow in the bottom left
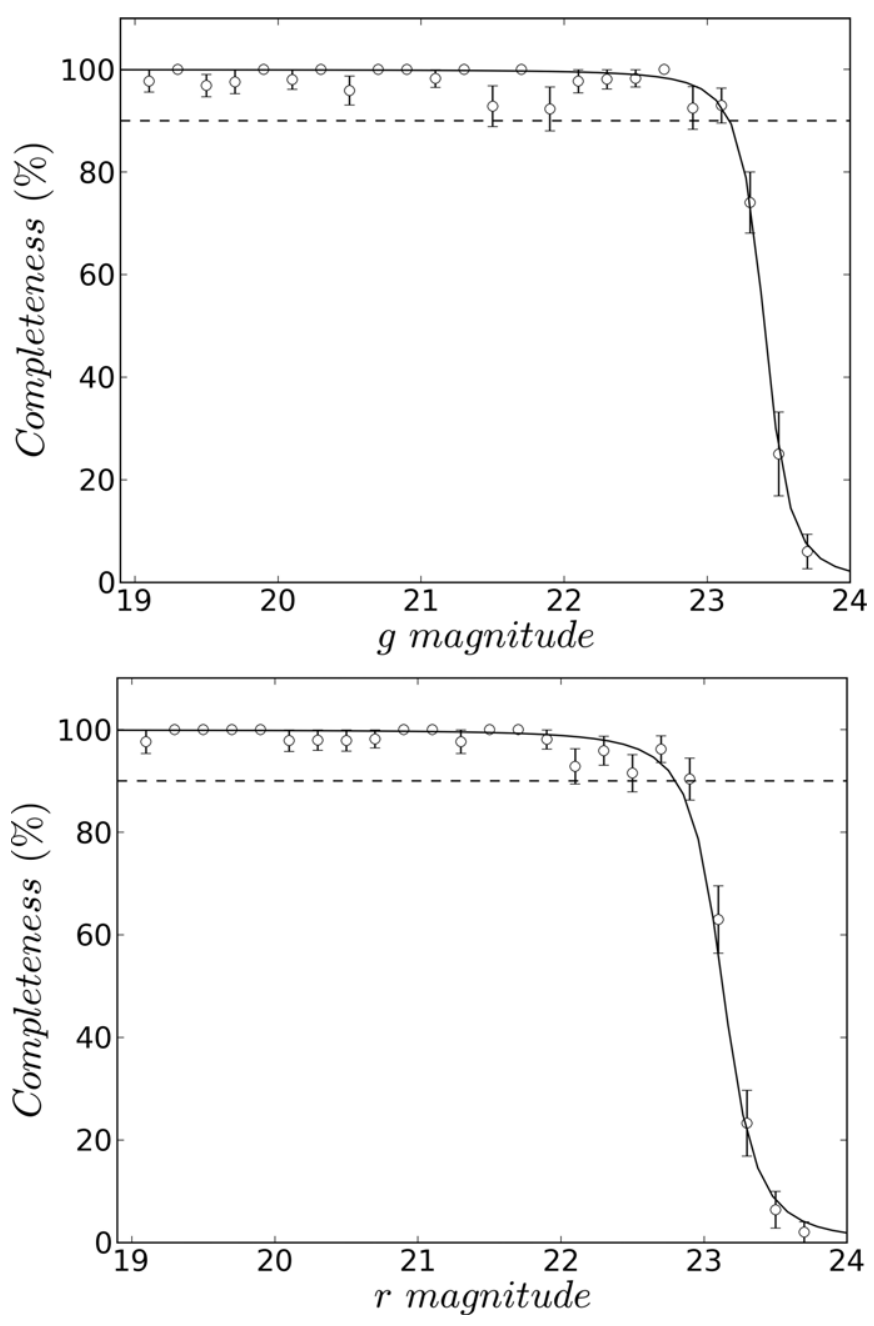

Figure 1. Artificial star tests: detection completeness as function of magnitude in the $g$ band (upper panel) and $r$ band (lower panel). Overplotted are the bestfitting analytic interpolation functions. The $90 \%$ completeness levels (dashed line) correspond to $g=23.15$ and $r=22.80$, respectively.

panel. These contiguous pixels are concentrated on the central region and there is no other comparable overdensity, in terms of both integrated signal and area, in the vicinity of Kim 1 at the same distance. The bottom right panel shows the corresponding contour plot, where the contours represent the different levels of normalized star density in units of standard deviation of the output signals above the Galactic foreground.

\section{CANDIDATE PROPERTIES}

\subsection{Color-Magnitude Diagram}

The upper left panel of Figure 3 shows the distribution of all objects classified as stars by the SDSS pipeline in the vicinity of Kim 1. For comparison we show the SDSS data for Segue 3 (Belokurov et al. 2010; Fadely et al. 2011) in the second row. Kim 1 has a lower star density and appears less prominent, until the photometric cut has been applied. The panels in the middle column of Figure 3 show the extinction-corrected CMDs of the two overdensities, and the panels in the right column that of the foreground stars around them. The CMDs of the foreground stars have been established following the same procedure as in Belokurov et al. $(2006,2007 b)$ from the area between two concentric rings covering the same area as Kim 1 and Segue 3, 

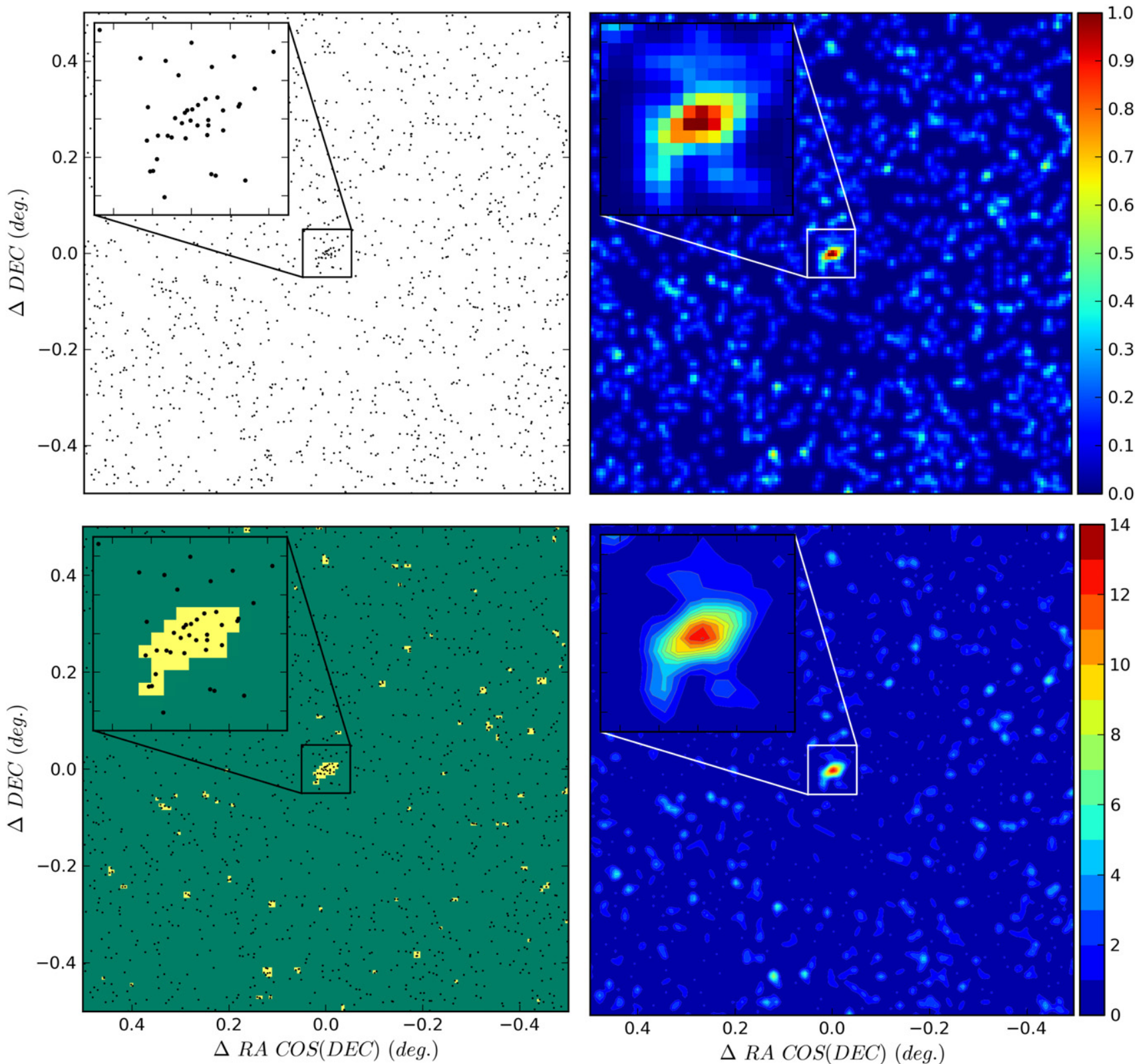

Figure 2. Upper left panel: spatial positions of DECam stars, in the magnitude range from the saturation level to the $90 \%$ completeness level for each $g$ and $r$ band, passing the photometric selection criteria. Upper right panel: binned spatial density with a pixel size of 23".4 and convolved with a Gaussian kernel with FWHM of $55^{\prime \prime} 1$. The color of the pixels represent the output signals $(s)$ of the convolution. Lower left panel: selected bins of which the output signals are above the foreground density $\tilde{s}+2.5 \sigma_{s} \approx 0.3$. Lower right panel: contour plot of the convolved density map. The contours show the levels of the output signals in units of $\sigma_{s}$ above the Galactic foreground.

respectively. Kim 1 has an equally well defined main sequence (MS) as Segue 3 down to $r_{0}=22 \mathrm{mag}$. There are also five stars with $r_{0}$ magnitudes brighter than 18 mag and colors consistent with red giant branch (RGB) and red clump stars. Finally, the panels in the bottom row of Figure 3 show the results for Kim 1 based on our DECam photometry reaching $\sim 2$ mag fainter than SDSS with $\mathrm{S} / \mathrm{N}$ values for point sources of 35 and 20, in $g$ and $r$, respectively, at 23rd AB mag. The DECam data reveals a tight main sequence between $19.5<r_{0}<24.0 \mathrm{mag}$.

\subsection{Age and Metallicity}

We estimate the age, $[\mathrm{Fe} / \mathrm{H}]$, and distance of Kim 1 using the maximum likelihood method described in Frayn \& Gilmore
(2002) and Fadely et al. (2011). We use all stars within a radius of $2^{\prime}$ around Kim 1, combining SDSS $(r<19)$ and DECam $(r>19)$ photometry. We calculate the maximum likelihood values $\mathcal{L}_{i}$ over a grid of Dartmouth model isochrones (Dotter et al. 2008) covering an age range from 7.0-13.5 Gyr, a metallicity range $-2.5 \leqslant[\mathrm{Fe} / \mathrm{H}] \leqslant-0.5 \mathrm{dex}$, and a distance range $16<(m-M)<17$. Grid steps are $0.5 \mathrm{Gyr}, 0.1 \mathrm{dex}$, and $0.05 \mathrm{mag}$. In Figure 4, we present the matrix of likelihood values for the sample described above after interpolation and smoothing over two grid points. We find the Dartmouth isochrone with the highest probability has an age of $12.0 \mathrm{Gyr}$ and $[\mathrm{Fe} / \mathrm{H}]=-1.7$ dex. The $68 \%, 95 \%$, and $99 \%$ confidence contours are also overplotted in the figure. The marginalized uncertainties about this most probable location correspond to an 

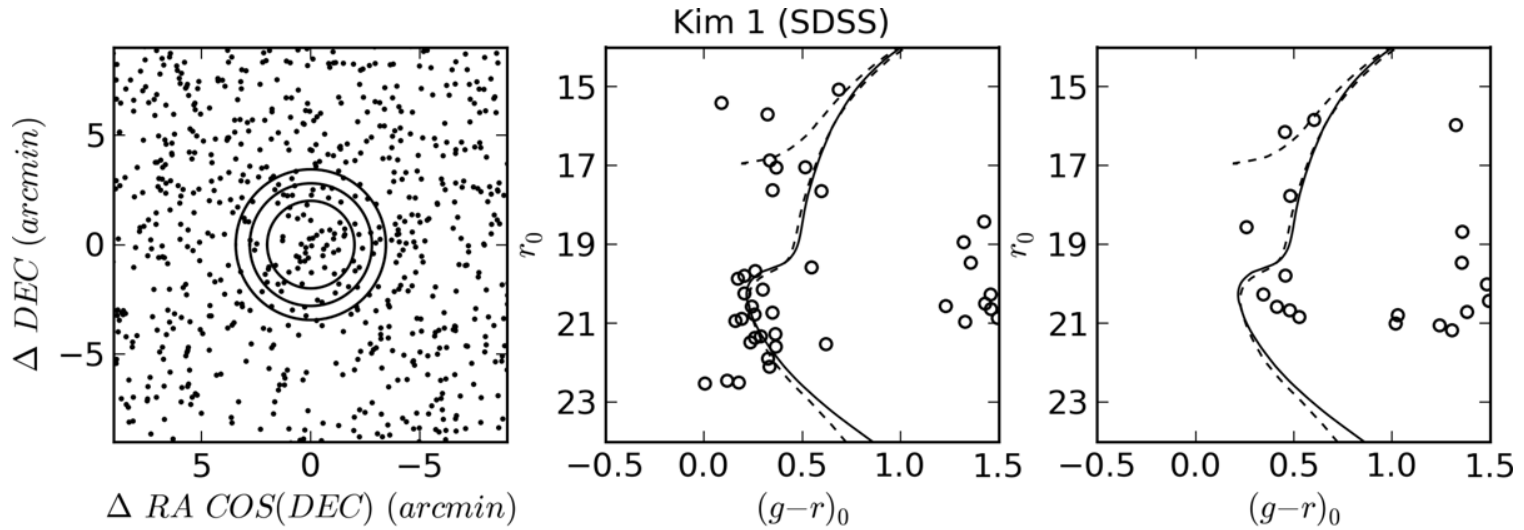

$\triangle R A C O S(D E C)($ arcmin $)$
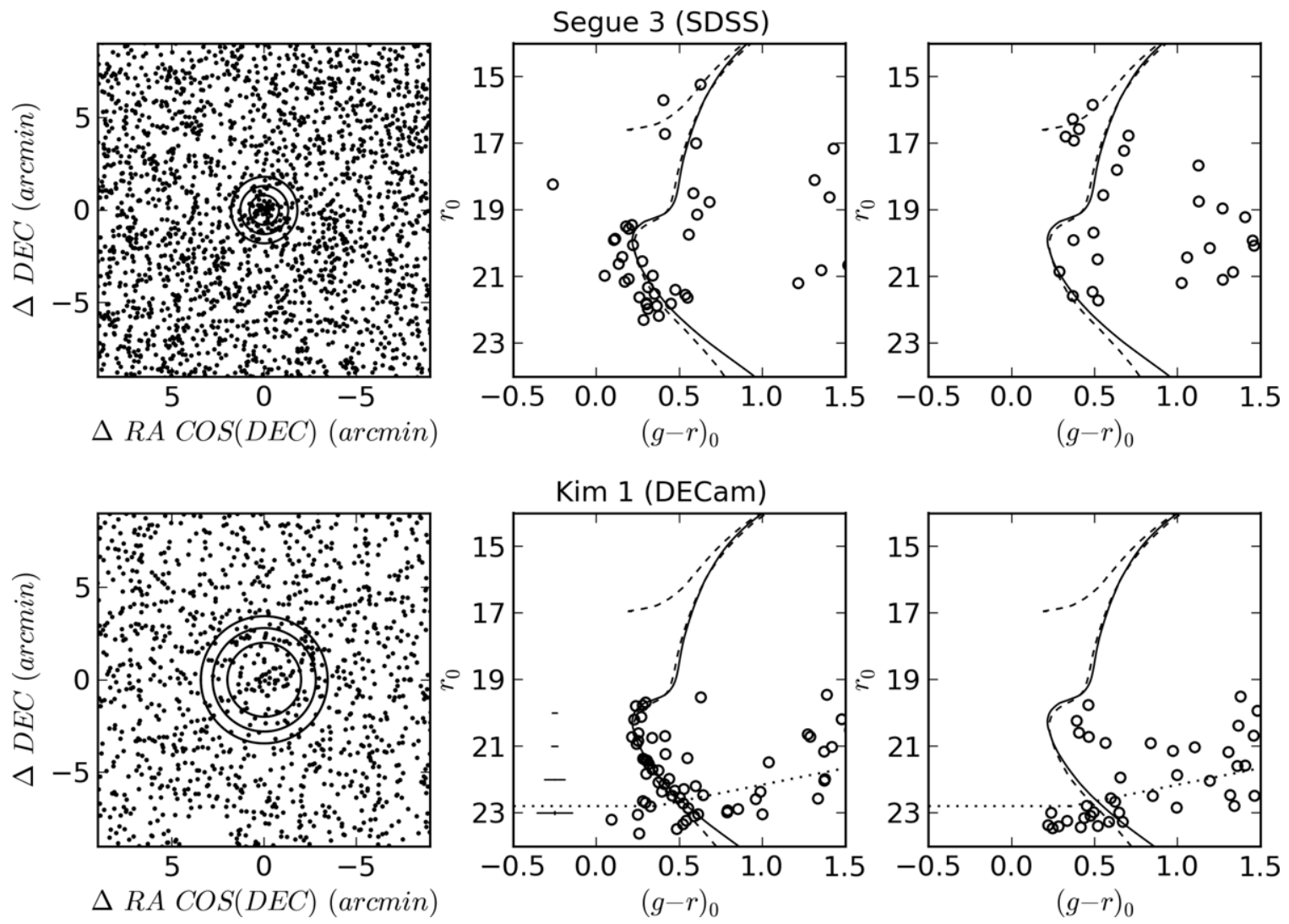

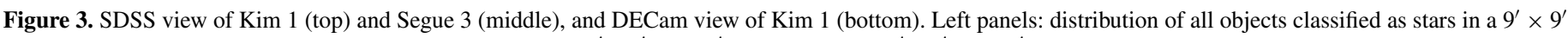

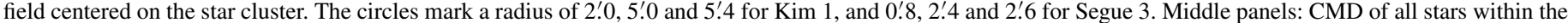

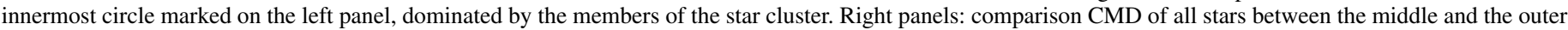

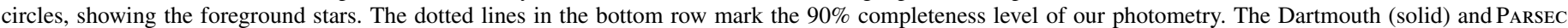

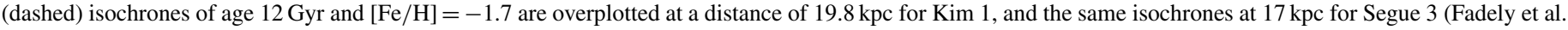
2011).

age of $12.0_{-3.0}^{+1.5} \mathrm{Gyr}$, a metallicity of $[\mathrm{Fe} / \mathrm{H}]=-1.7_{-0.2}^{+0.5} \mathrm{dex}$, and a distance modulus of $(\mathrm{m}-\mathrm{M})_{0}=16.48 \pm 0.10 \mathrm{mag}$ $\left(d_{\odot}=19.8 \pm 0.9 \mathrm{kpc}\right)$. We adopt a heliocentric distance of $19.8 \mathrm{kpc}$ in the calculation of physical size and absolute magnitude in Section 4.2.

The best-fitting Dartmouth and PARSEC (Bressan et al. 2012) isochrones of age $12 \mathrm{Gyr}$ and $[\mathrm{Fe} / \mathrm{H}]=-1.7$ that match the location of the RGB, red clump and MS of Kim 1 are overplotted at a distance of $19.8 \mathrm{kpc}$ to illustrate the consistency with an old metal-poor stellar population independently of the theoretical model. Segue 3 happens to have the same age and metallicity (Fadely et al. 2011). We adopted a distance of $17 \mathrm{kpc}$ for the isochrone shown in the CMD of Segue 3.

\subsection{Size, Ellipticity, and Luminosity}

Figure 5 shows the $6 \times 6 \operatorname{arcmin}^{2}$ DECam cutout $g$-band image of Kim 1. The circles indicate the locations of all stars within $2 r_{h}$ from Kim 1 in Figure 3 that passed our photometric cut based on the isochrone fit in the color-magnitude space.

The left panel of Figure 6 shows the sky distribution of all DECam stars, in the magnitude range from the saturation level to the $90 \%$ completeness level for each $g$ and $r$ band, passing the photometric cut based on the best-fitting model isochrone, in a $0.3 \times 0.3$ window centered on Kim 1 . The right panel shows the associated radial profile of the stellar number density, i.e., star counts in elliptical annuli around Kim 1, where $r$ is the elliptical 


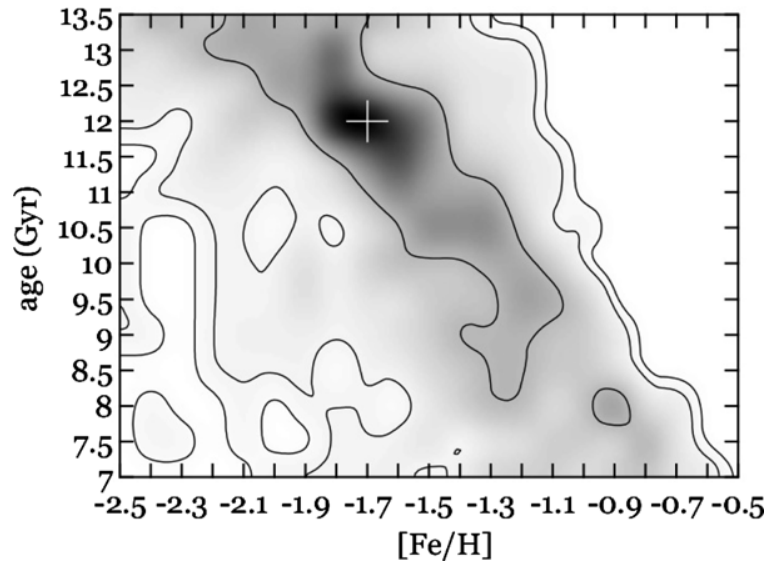

Figure 4. Smoothed maximum likelihood density map in age-metallicity space for all stars within a radius of $2^{\prime}$ around Kim 1 , combined with SDSS $(r<19)$ and DECam $(r>19)$ coverage. Contour lines show the 68\%,95\%, and $99 \%$ confidence levels. The diagonal flow of the contour lines reflect the age-metallicity degeneracy inherent to such an isochrone fitting procedure The 1D marginalized parameters around the best fit are: age $=12.0_{-3.0}^{+1.5} \mathrm{Gyr}$, $[\mathrm{Fe} / \mathrm{H}]=-1.7_{-0.2}^{+0.6} \mathrm{dex}, m-M=16.48 \pm 0.10 \mathrm{mag}$.

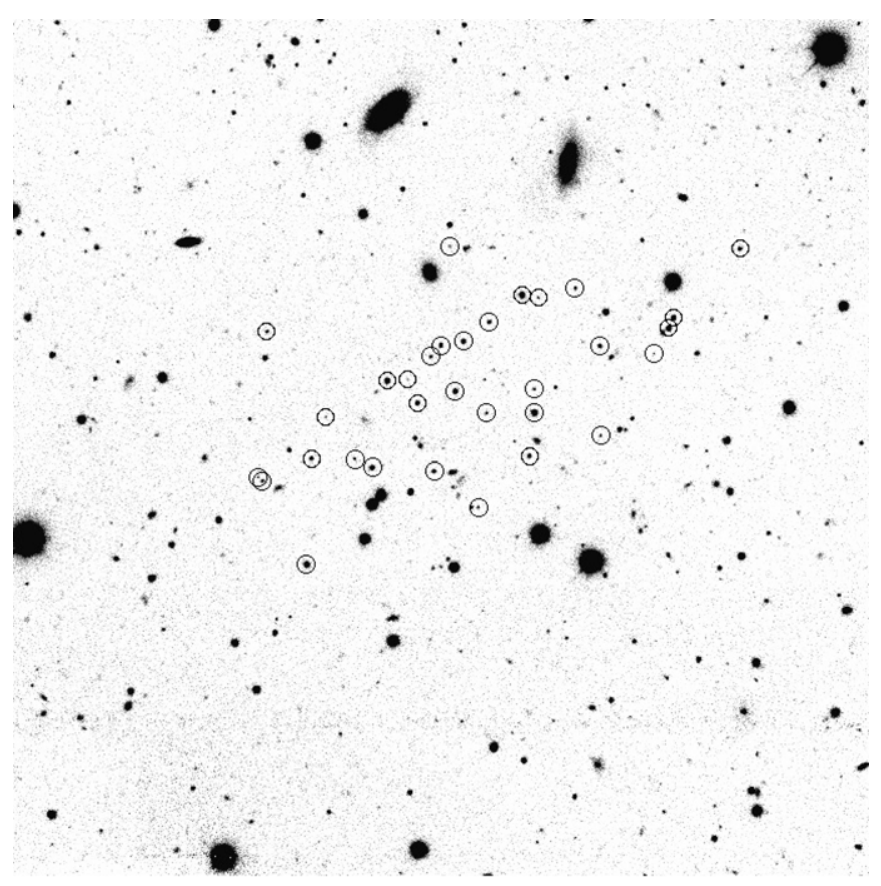

Figure 5. $6 \times 6 \operatorname{arcmin}^{2}$ DECam cutout $g$-band image of Kim 1 . North is up, east is to the left. Circled are all stars within $2 r_{h}$ from Kim 1 that are consistent in color-magnitude space with the best-fitting isochrone $(12 \mathrm{Gyr},[\mathrm{Fe} / \mathrm{H}]=-1.7)$ at a distance of $19.8 \mathrm{kpc}$. The main-sequence stars are fainter than $r_{0} \approx 19.5$ and have colors within $0.1<(g-r)_{0}<0.6$.

radius. For the center of the overdensity, we adopted the center of mass calculated in terms of the normalized signals on the array into which the stars were binned and smoothed as described in Section 2. We derive an ellipticity $\epsilon=0.42 \pm 0.10$ and a position angle $\theta=-59 \pm 6$ deg by using the fit_bivariate_normal function of the astroML package (VanderPlas et al. 2012). The error bars were derived from Poisson statistics. Overplotted is the best-fit Plummer profile (Plummer 1911) to parameterize the underlying stellar distribution. We obtain a half-light radius of $1.2 \pm 0.1$ arcmin or $r_{h}=6.9 \pm 0.6 \mathrm{pc}$, adopting the distance modulus of $16.48 \mathrm{mag}$.
Table 1

Properties of Kim 1

\begin{tabular}{lcc}
\hline \hline Parameter & Value & Unit \\
\hline$\alpha_{J 2000}$ & $22: 11: 41.3$ & $\mathrm{~h}: \mathrm{m}: \mathrm{s}$ \\
$\delta_{J 2000}$ & $+07: 01: 31.8$ & $\circ:^{\prime}:^{\prime \prime}$ \\
$l$ & 68.5148 & $\mathrm{deg}$ \\
$b$ & -38.4256 & $\mathrm{deg}$ \\
$(m-M)$ & $16.48 \pm 0.10$ & $\mathrm{mag}$ \\
$d_{\odot}$ & $19.8 \pm 0.9$ & $\mathrm{kpc}$ \\
{$[\mathrm{Fe} / \mathrm{H}]$} & $-1.7_{-0.2}^{+0.5}$ & $\mathrm{dex}$ \\
$\mathrm{Age}$ & $12_{-3.0}^{+1.5}$ & $\mathrm{Gyr}$ \\
$r_{h}(\mathrm{Plummer})$ & $6.9 \pm 0.6^{\mathrm{a}}$ & $\mathrm{pc}$ \\
$\epsilon$ & $0.42 \pm 0.10$ & $\mathrm{deg}$ \\
$\theta$ & $-59 \pm 6$ & $\mathrm{mag}$ \\
$M_{\mathrm{tot}, \mathrm{V}}$ & $0.3 \pm 0.5$ & \\
\hline
\end{tabular}

Note. ${ }^{a}$ Adopting a distance of $19.8 \mathrm{kpc}$.

In analogy to Walsh et al. (2008), we estimate the total luminosity of Kim 1 by integrating the radial number density profile shown in Figure 6 to calculate the total number of Kim 1 stars $N_{*}$ within the photometric limits. Using the ratio of this number $N_{*}$ to the probability density of a normalized theoretical luminosity function (LF) in the same magnitude range as for the radial profile, we scale the theoretical LF to the number densities of stars as a function of $r$ magnitude. Integrating the scaled LF inclusive of the missing flux from stars brighter than the saturation limit and fainter than the $90 \%$ completeness limit, gives a total luminosity $M_{r, \operatorname{Kim} 1}=0.04 \mathrm{mag}$ based on the PARSEC isochrone and $M_{r, \operatorname{Kim} 1}=-0.07 \mathrm{mag}$ based on the Dartmouth isochrone, both for a $12 \mathrm{Gyr}$ old stellar population with $[\mathrm{Fe} / \mathrm{H}]=-1.7$. Due to the intrinsic faintness of the stellar overdensity and the low number of stars, the estimates of the total luminosity has a large uncertainty. From the $V-r$ colors $(0.32 \mathrm{mag}$ and $0.22 \mathrm{mag})$ of the two best-fitting model isochrones we derive the corresponding $V$-band luminosities $M_{V, \operatorname{Kim} 1}=0.36 \mathrm{mag}$ and $M_{V, \operatorname{Kim} 1}=0.15 \mathrm{mag}$, respectively. We note that both model isochrones give consistent results. Since this method relies on the number of stars in such an ultrafaint stellar system instead of their individual flux, the inclusion or exclusion of a single RGB or red clump star in the system can change its total luminosity still by $\sim 0.5 \mathrm{mag}$. Hence, a realistic estimate of the total luminosity of Kim 1 is $M_{V}=0.3 \pm 0.5$. All derived parameters are summarized in Table 1.

\section{DISCUSSION AND CONCLUSION}

We report the new ultra-faint stellar overdensity, Kim 1, in the constellation of Pegasus. The best-fitting theoretical isochrone reveals a single $12 \mathrm{Gyr}$ old, metal-poor $([\mathrm{Fe} / \mathrm{H}]=-1.7)$ stellar population at a well-defined heliocentric distance of $19.8 \pm$ $0.9 \mathrm{kpc}$. Its total luminosity of $M_{V}=0.3 \pm 0.5$ shows that Kim 1 is among the faintest stellar systems discovered in the MW halo to date. Other MW star clusters known to have comparable luminosities are Segue $3\left(M_{V}=0.0 \pm 0.8\right.$; Fadely et al. 2011), Muñoz $1\left(M_{V}=-0.4 \pm 0.9\right.$; Muñoz et al. 2012), Balbinot 1 $\left(M_{V}=-1.21 \pm 0.66\right.$; Balbinot et al. 2013), and Koposov 1 and $2\left(M_{V}=-2, M_{V}=-1\right.$; Koposov et al. 2007). Its physical size $\left(r_{h}=6.9 \pm 0.6 \mathrm{pc}\right)$ and low luminosity place Kim 1 close to these MW star clusters in the size-luminosity diagram (Fadely et al. 2011). 

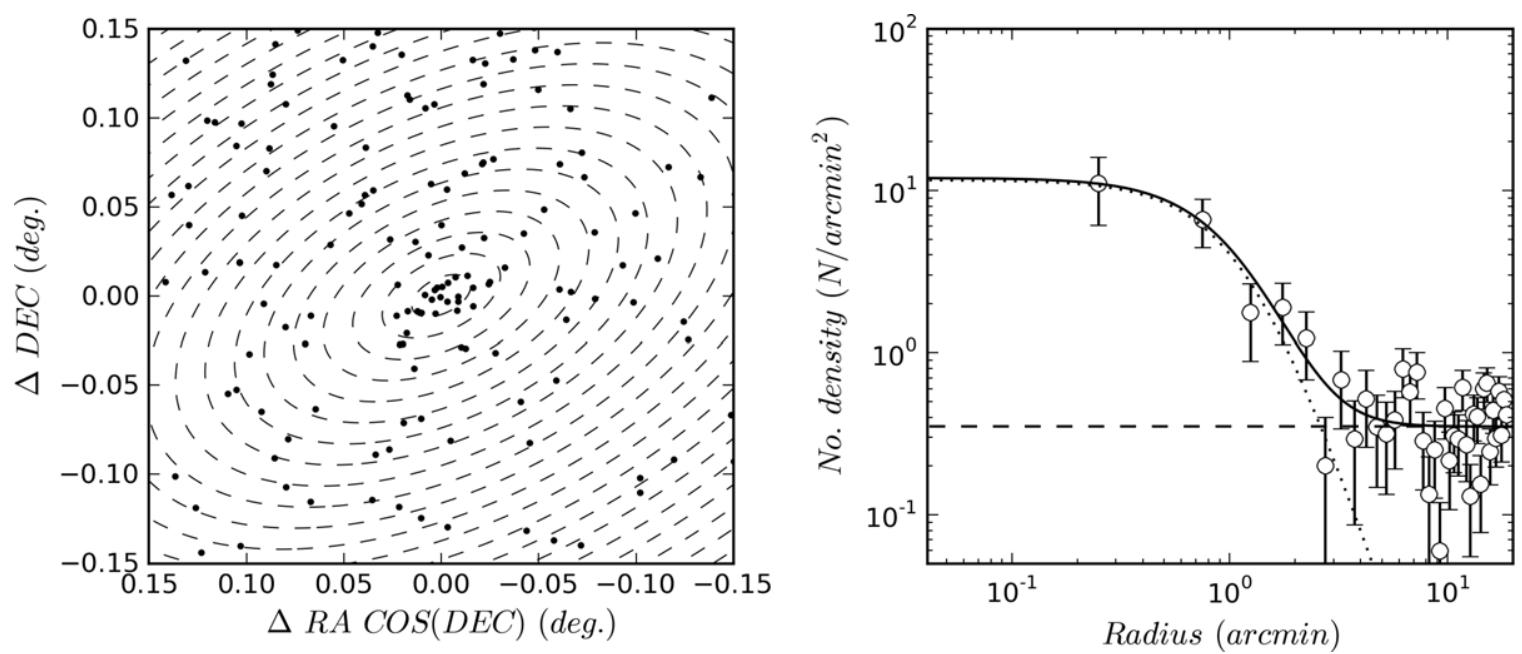

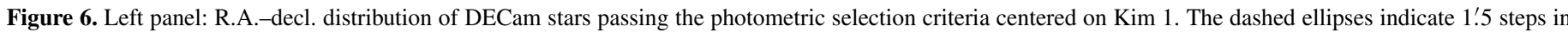

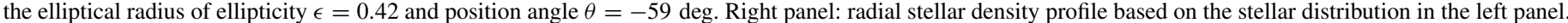

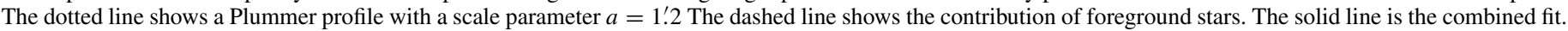

\subsection{Kim 1, a Dissolving Star Cluster?}

Despite the fact that the stellar population of Kim 1 appears sufficiently old to be dynamically relaxed for the given physical size and the number of constituent stars, the overdensity has an unusually high ellipticity of 0.42 and a poorly defined central concentration. No other low-luminosity MW star cluster, with the exception of Koposov 1 (Koposov et al. 2007, see their Figure 2(a)) has comparable structural properties, which suggests that we are seeing a tidally disrupted star cluster or a remnant thereof. We note that the small size of Kim 1 and the absence of a more complex stellar population make it unlikely that Kim 1 is a ultra-faint satellite galaxy of the MW (Willman \& Strader 2012).

Although a detailed simulation of Kim 1's dynamical history is beyond the scope of this study due to the lack of kinematical data and orbital parameters, H. Baumgardt (2014, private communication) kindly ran $N$-body simulations using NBODY6 (Aarseth 1999) to estimate the initial mass of the progenitor star cluster, using a setup similar to Baumgardt \& Makino (2003). For that purpose, a model star cluster with a Kroupa (2001) initial mass function was placed on circular or eccentric orbits in a spherical MW potential with a constant rotation velocity of $V_{G}=220 \mathrm{~km} \mathrm{~s}^{-1}$. The integration of the orbits was halted after $12 \mathrm{Gyr}$, the age of Kim 1. Matching the bound mass of the remnant model star cluster with the current mass of Kim 1 (approximately $100 M_{\odot}$ ) confines its initial stellar mass to the range $3500 M_{\odot}<M_{\text {init }}<5500 M_{\odot}$, where the two limits correspond to a highly eccentric orbit with a perigalactic and apogalactic distance of $20 \mathrm{kpc}$ and $100 \mathrm{kpc}$, respectively, and a circular orbit at the distance of $25 \mathrm{kpc}$. In Figure 7, we show the line-of-sight stellar distribution of the model star cluster in the circular orbit after $12 \mathrm{Gyr}$ for the DECam field of view (left) as well as the inner region (right). There are only a small number of extra tidal stars distributed over an area corresponding to the DECam field, and the remnant star cluster is highly asymmetric and elongated in the inner $30 \mathrm{pc} \times 30 \mathrm{pc}$ without a well-defined center. From these simulations we can conclude that the observational properties of Kim 1 are consistent with a dissolving star cluster that initially had a few thousand solar masses. No prominent stellar stream is expected to be associated to such a low-mass star cluster. The few cluster stars found beyond the tidal radius would be completely hidden in the screen of stars from the MW.

\subsection{Is Kim 1 Associated to a Stream?}

The constellation of Pegasus, in which Kim 1, Segue 3 and Balbinot 1 are found, lies close to the Hercules-Aquila Cloud discovered in SDSS-DR5 (Belokurov et al. 2007a). This Galactic halo substructure is centered at $l \sim 40^{\circ}$, and extends down to $b \sim-40^{\circ}$ at $l \sim 50^{\circ}$. In fact, de Jong et al. (2010) used main-sequence stars in SDSS-DR7 SEGUE data to identify a number of new halo overdensities that might be related to the cloud. As Segue 3 coincides spatially with one of the reported overdensities, there has been speculations about the possible association of Segue 3 with the Hercules-Aquila Cloud. A spectroscopic analysis (Fadely et al. 2011), however, found a kinematic offset that suggests Segue 3 is unlikely associated with that overdensity. For Balbinot 1 and Kim 1, located at $(l, b)=(75.1735,-32.6432)$ and $(l, b)=(68.5148$, $-34.4256)$, respectively, there is no corresponding overdensity out of all the detections listed in Table 4 of de Jong et al. (2010). In addition, there is an argument that the true heliocentric distance of the Hercules-Aquila Cloud ranges only between 1 and $6 \mathrm{kpc}$ (Larsen et al. 2011). Simion et al. (2014) conclude in their recent study using RR Lyrae stars in the Catalina Sky Survey data that the extension of the Hercules-Aquila Cloud is bound within $30^{\circ}<l<55^{\circ}$ and $-45^{\circ}<b<-25^{\circ}$, and that there is no significant excess of stars in the region of sky at $55^{\circ}<l<85^{\circ}$ and $-45^{\circ}<b<-25^{\circ}$. Since Kim 1, Segue 3, and Balbinot 1 are all located at $l>68^{\circ}$, these star clusters are unlikely associated with the Hercules-Aquila Cloud.

Recent searches of SDSS DR8 and DR10 for extended stellar overdensities in the Galactic halo have discovered new stellar streams in a range of heliocentric distances from 15 to $35 \mathrm{kpc}$ (Bonaca et al. 2012; Martin et al. 2013; Grillmair 2014). These stellar streams are identified on extended stellar density maps by taking photometric cuts with weighted isochrone masks over a range of distance moduli. Due to the sparse and extended nature of the streams, their stellar populations are barely discerned from the MW foreground in color-magnitude space unless a fieldsubtracted Hess diagram is constructed out of a sufficiently large sky area. None of those searches, however, have found any stellar 

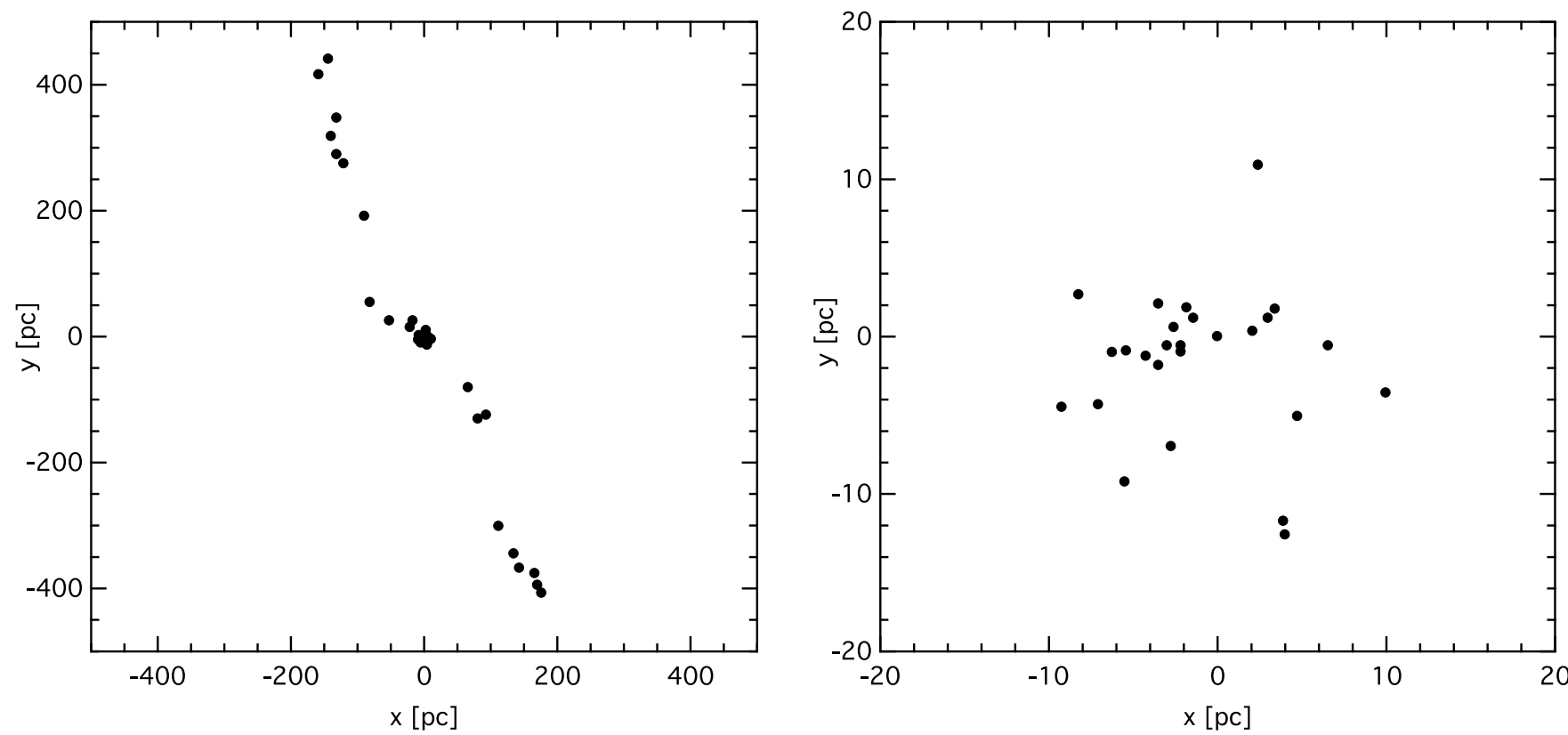

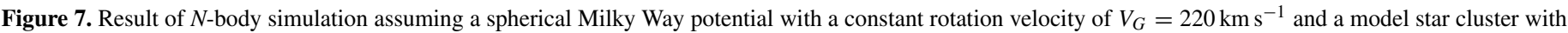

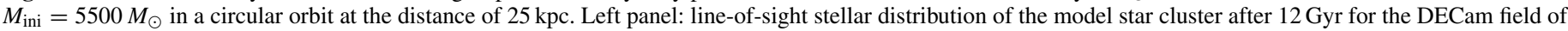
view. Right panel: the same as the left panel but for the inner region. Star counts and stellar distribution closely resemble Kim 1.

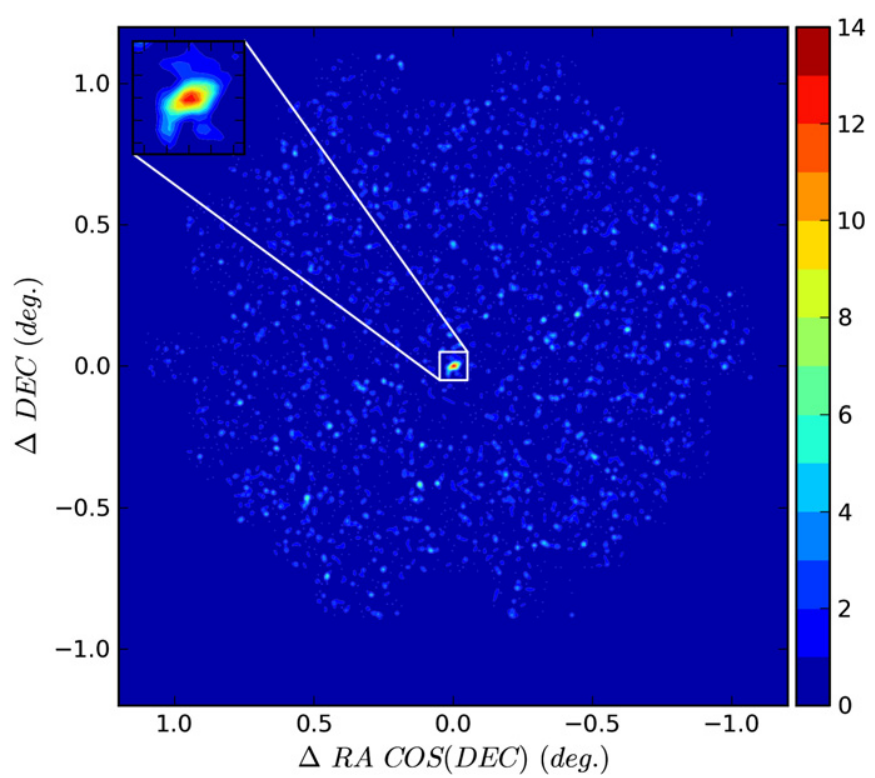

Figure 8. Same as the lower right panel of Figure 2 but for all stars in the field of view of DECam.

stream that overlaps with the location of Kim 1 (e.g., Figure 2 in Bonaca et al. 2012), and the stellar population of Kim 1 is clearly distinguished from the foreground in the CMD of a localized region. In addition, unlike tidal debris detected around Pal 5 (Odenkirchen et al. 2001), our DECam star distribution does not feature any extra significant clumps around Kim 1 out to $400 \mathrm{pc}$ radius. There is also a hypothesis that ultra-faint dwarf galaxies are instead cusp caustics of cold stellar debris that formed during the disruption of low-mass satellites (Zolotov et al. 2011). A cusp caustic in projection appears as a highly elongated center of stars, along with a twofold of tails as an indication. Although Kim 1 indeed appears highly elongated compared to other known ultra-faint star clusters, such small-scale tails and their fold are not detected in our DECam data (see Figure 8).
It is worth noticing that Segue $3\left(d_{\odot}=17 \mathrm{kpc}, 12 \mathrm{Gyr}\right.$, [Fe/ $\mathrm{H}]=-1.7), \operatorname{Kim} 1\left(d_{\odot}=19.8 \mathrm{kpc}, 12 \mathrm{Gyr},[\mathrm{Fe} / \mathrm{H}]=-1.7\right)$, and Balbinot $1\left(d_{\odot}=31.9 \mathrm{kpc}, 11.7 \mathrm{Gyr},[\mathrm{Fe} / \mathrm{H}]=-1.58\right)$, are neighbors in projection and even lie within a considerably narrow range of heliocentric distances from 17 to $32 \mathrm{kpc}$. They are also spatially close to the globular cluster $\mathrm{M} 2\left(d_{\odot}=11.5 \mathrm{kpc}\right.$; Harris 1996), one of the most unusual globular clusters in the MW halo with a number of distinct stellar subpopulations (Milone et al. 2014), and their locations coincide with the vast polar structure (VPOS), a thin $(20 \mathrm{kpc})$ plane perpendicular to the MW disk defined by the 11 brightest MW satellite galaxies (Kroupa et al. 2005; Metz et al. 2007, 2009; Kroupa et al. 2010; Pawlowski et al. 2012). Globular clusters and stellar and gaseous streams appear to preferentially aligned with the VPOS too (Forbes et al. 2009; Pawlowski et al. 2012). It might be a coincidence that all four stellar systems mentioned above are spatially close together and that their locations agree with the VPOS, but this finding naturally raises the question about these star clusters' possible link to the MW satellite galaxies. While more information is required to address Kim 1's origin, the finding of such a small, extreme low-luminosity stellar concentration in the inner halo is definitely consistent with current detection limits (Walsh et al. 2009, see Figure 15) and points to a much larger parent population of star clusters that are still undetected in the MW halo or must have been already destroyed by the Galactic tidal field.

The authors thank Tammy Roderick, Kathy Vivas, and David James for their assistance during the DECam observing run. We gratefully acknowledge Holger Baumgardt for producing the $N$-body results described in the discussion. We also thank the referee for the helpful comments and suggestions, which contributed to improving the quality of the publication. We acknowledge the support of the Australian Research Council (ARC) through Discovery project DP120100475 and financial support from the Go8/Germany Joint Research Co-operation Scheme. Funding for SDSS-III has been provided by the Alfred 
P. Sloan Foundation, the Participating Institutions, the National Science Foundation, and the U.S. Department of Energy Office of Science. The SDSS-III Web site is http://www.sdss3.org/.

SDSS-III is managed by the Astrophysical Research Consortium for the Participating Institutions of the SDSS-III Collaboration including the University of Arizona, the Brazilian Participation Group, Brookhaven National Laboratory, Carnegie Mellon University, University of Florida, the French Participation Group, the German Participation Group, Harvard University, the Instituto de Astrofisica de Canarias, the Michigan State/ Notre Dame/JINA Participation Group, Johns Hopkins University, Lawrence Berkeley National Laboratory, Max Planck Institute for Astrophysics, Max Planck Institute for Extraterrestrial Physics, New Mexico State University, New York University, Ohio State University, Pennsylvania State University, University of Portsmouth, Princeton University, the Spanish Participation Group, University of Tokyo, University of Utah, Vanderbilt University, University of Virginia, University of Washington, and Yale University.

This project used data obtained with the Dark Energy Camera (DECam), which was constructed by the Dark Energy Survey (DES) collaborating institutions: Argonne National Lab, University of California Santa Cruz, University of Cambridge, Centro de Investigaciones Energeticas, Medioambientales y Tecnologicas-Madrid, University of Chicago, University College London, DES-Brazil consortium, University of Edinburgh, ETH-Zurich, University of Illinois at Urbana-Champaign, Institut de Ciencies de l'Espai, Institut de Fisica d'Altes Energies, Lawrence Berkeley National Lab, Ludwig-Maximilians Universitat, University of Michigan, National Optical Astronomy Observatory, University of Nottingham, Ohio State University, University of Pennsylvania, University of Portsmouth, SLAC National Lab, Stanford University, University of Sussex, and Texas A\&M University. Funding for DES, including DECam, has been provided by the U.S. Department of Energy, National Science Foundation, Ministry of Education and Science (Spain), Science and Technology Facilities Council (UK), Higher Education Funding Council (England), National Center for Supercomputing Applications, Kavli Institute for Cosmological Physics, Financiadora de Estudos e Projetos, Fundação Carlos Chagas Filho de Amparo a Pesquisa, Conselho Nacional de Desenvolvimento Científico e Tecnológico and the Ministério da Ciência e Tecnologia (Brazil), the German Research Foundation-sponsored cluster of excellence "Origin and Structure of the Universe" and the DES collaborating institutions.

\section{REFERENCES}

Aarseth, S. J. 1999, PASP, 111, 1333

Ahn, C. P., Alexandroff, R., Allende Prieto, C., et al. 2014, ApJS, 211, 17 Balbinot, E., Santiago, B. X., da Costa, L., et al. 2013, ApJ, 767, 101 Baumgardt, H., \& Makino, J. 2003, MNRAS, 340, 227

Belokurov, V., Evans, N. W., Bell, E. F., et al. 2007a, ApJL, 657, L89 Belokurov, V., Irwin, M. J., Koposov, S. E., et al. 2014, MNRAS, 441, 2124 Belokurov, V., Walker, M. G., Evans, N. W., et al. 2010, ApJL, 712, L103 Belokurov, V., Zucker, D. B., Evans, N. W., et al. 2006, ApJL, 642, L137
Belokurov, V., Zucker, D. B., Evans, N. W., et al. 2007b, ApJ, 654, 897

Bertin, E., \& Arnouts, S. 1996, A\&AS, 117, 393

Bolte, M. 1989, ApJ, 341, 168

Bonaca, A., Geha, M., \& Kallivayalil, N. 2012, ApJL, 760, L6

Bressan, A., Marigo, P., Girardi, L., et al. 2012, MNRAS, 427, 127 Carraro, G. 2009, AJ, 137, 3809

Carraro, G., Zinn, R., \& Moni Bidin, C. 2007, A\&A, 466, 181

de Jong, J. T. A., Yanny, B., Rix, H.-W., et al. 2010, ApJ, 714, 663

Dotter, A., Chaboyer, B., Jevremović, D., et al. 2008, ApJS, 178, 89

Fadely, R., Willman, B., Geha, M., et al. 2011, AJ, 142, 88

Fleming, D. E. B., Harris, W. E., Pritchet, C. J., \& Hanes, D. A. 1995, AJ, 109,1044

Forbes, D. A., Kroupa, P., Metz, M., \& Spitler, L. 2009, arXiv:0906.5370

Frayn, C. M., \& Gilmore, G. F. 2002, MNRAS, 337, 445

Gnedin, O. Y., \& Ostriker, J. P. 1997, ApJ, 474, 223

Grillmair, C. J. 2014, ApJL, 790, L10

Harris, W. E. 1996, AJ, 112, 1487

Irwin, M. J., Belokurov, V., Evans, N. W., et al. 2007, ApJL, 656, L13

Kirby, E. N., Cohen, J. G., Guhathakurta, P., et al. 2013, ApJ, 779, 102

Kirby, E. N., Simon, J. D., Geha, M., Guhathakurta, P., \& Frebel, A. 2008, ApJL, 685, L43

Koch, A., \& Rich, R. M. 2014, ApJ, 794, 89

Koposov, S., Belokurov, V., Evans, N. W., et al. 2008, ApJ, 686, 279

Koposov, S., de Jong, J. T. A., Belokurov, V., et al. 2007, ApJ, 669, 337

Kroupa, P. 2001, MNRAS, 322, 231

Kroupa, P., Famaey, B., de Boer, K. S., et al. 2010, A\&A, 523, A32

Kroupa, P., Theis, C., \& Boily, C. M. 2005, A\&A, 431, 517

Laevens, B. P. M., Martin, N. F., Sesar, B., et al. 2014, ApJL, 786, L3

Larsen, J. A., Cabanela, J. E., \& Humphreys, R. M. 2011, AJ, 141, 130

Marmo, C., \& Bertin, E. 2008, in ASP Conf. Ser. 394, Astronomical Data Analysis Software and Systems XVII, ed. R. W. Argyle, P. S. Bunclark, \& J. R. Lewis (San Francisco, CA: ASP), 619

Martin, C., Carlin, J. L., Newberg, H. J., \& Grillmair, C. 2013, ApJL, 765, L39 Martin, N. F., de Jong, J. T. A., \& Rix, H.-W. 2008, ApJ, 684, 1075

Metz, M., Kroupa, P., \& Jerjen, H. 2007, MNRAS, 374, 1125

Metz, M., Kroupa, P., \& Jerjen, H. 2009, MNRAS, 394, 2223

Milone, A. P., Marino, A. F., Piotto, G., et al. 2014, MNRAS, in press (arXiv:1411.5043)

Muñoz, R. R., Geha, M., Côté, P., et al. 2012, ApJL, 753, L15

Niederste-Ostholt, M., Belokurov, V., Evans, N. W., et al. 2010, MNRAS, 408, L66

Norris, J. E., Yong, D., Gilmore, G., \& Wyse, R. F. G. 2010, ApJ, 711, 350

Odenkirchen, M., Grebel, E. K., Rockosi, C. M., et al. 2001, ApJL, 548, L165

Pawlowski, M. S., Pflamm-Altenburg, J., \& Kroupa, P. 2012, MNRAS, 423, 1109

Plummer, H. C. 1911, MNRAS, 71, 460

Rosenberg, A., Saviane, I., Piotto, G., Aparicio, A., \& Zaggia, S. R. 1998, AJ, 115,648

Schlegel, D. J., Finkbeiner, D. P., \& Davis, M. 1998, ApJ, 500, 525

Simion, I. T., Belokurov, V., Irwin, M., \& Koposov, S. E. 2014, MNRAS, 440,161

Simon, J. D., Geha, M., Minor, Q. E., et al. 2011, ApJ, 733, 46

Taylor, M. B. 2006, in ASP Conf. Ser. 351, Astronomical Data Analysis Software and Systems XV, ed. C. Gabriel et al. (San Francisco, CA: ASP), 666

Valdes, F., Gruendl, R., \& DES Project, 2014, ASP Conf. Ser. 485, Astronomical Data Analysis Software and Systems XXIII, ed. N. Manset \& P. Forshay (San Francisco, CA: ASP), 379

VanderPlas, J., Connolly, A. J., Ivezic, Z., \& Gray, A. 2012, in Proceedings of Conference on Intelligent Data Understanding (CIDU), ed. K. Das, N. V. Chawla, \& N. Srivastava (Los Alamitos, CA: IEEE), 47

Walsh, S. M., Jerjen, H., \& Willman, B. 2007, ApJL, 662, L83

Walsh, S. M., Willman, B., \& Jerjen, H. 2009, AJ, 137, 450

Walsh, S. M., Willman, B., Sand, D., et al. 2008, ApJ, 688, 245

Willman, B., Blanton, M. R., West, A. A., et al. 2005, AJ, 129, 2692

Willman, B., \& Strader, J. 2012, AJ, 144, 76

York, D. G., Adelman, J., Anderson, J. E., Jr., et al. 2000, AJ, 120, 1579

Zolotov, A., Hogg, D. W., \& Willman, B. 2011, ApJL, 727, L14 